\title{
Idiopathic premature ventricular contractions originating from left ventricular summit successfully ablated from the epicardial approach
}

\author{
Tolga Aksu', Tümer Erdem Güler ${ }^{1}$, Serdar Bozyel ${ }^{1}$
}

1) University of Health Sciences, Kocaeli Derince Training and Research Hospital, Department of Cardiology, Kocaeli, Turkey

\begin{abstract}
Catheter ablation of ventricular arrhythmias originating from the left ventricular summit can be challenging, given the high risk of vascular injury and low success rates during radiofrequency ablation. In case of failed endocardial ablation attempt, ablation from adjacent structures such as the coronary cusps, coronary venous system, and the septal right ventricular outflow tract should be tried as effective alternative approaches, respectively. Epicardial approach is an effective alternative strategy in failed cases. We report on a 45-year-old man with VAs originated from the LVS who underwent an epicardial ablation.
\end{abstract}

Keywords: Epicardial ablation, vascular injury, ventricular arrhythmy, venous system.

Aksu T., Güler T. E., Bozyel S.: Idiopathic Premature Ventricular Contractions Originating From Left Ventricular Summit Successfully Ablated From The Epicardial Approach EJCM 2018; 06 (3): 117-121. Doi: 10.32596/ejcm.18.00317. 


\section{Introduction}

Idiopathic ventricular arrhythmias (VAs) are usually originated from outflow tract region of right and left ventricles, respectively.(1) The left ventricular summit (LVS) is a special triangular region in the epicardial left ventricular outflow tract bounded by the bifurcation between the left anterior descending and the left circumflex coronary arteries where the great cardiac vein ends and the anterior interventricular cardiac vein begins. ${ }^{(2)}$ Due to close proximity to major coronary vessels and

Figure 2. A,B,C

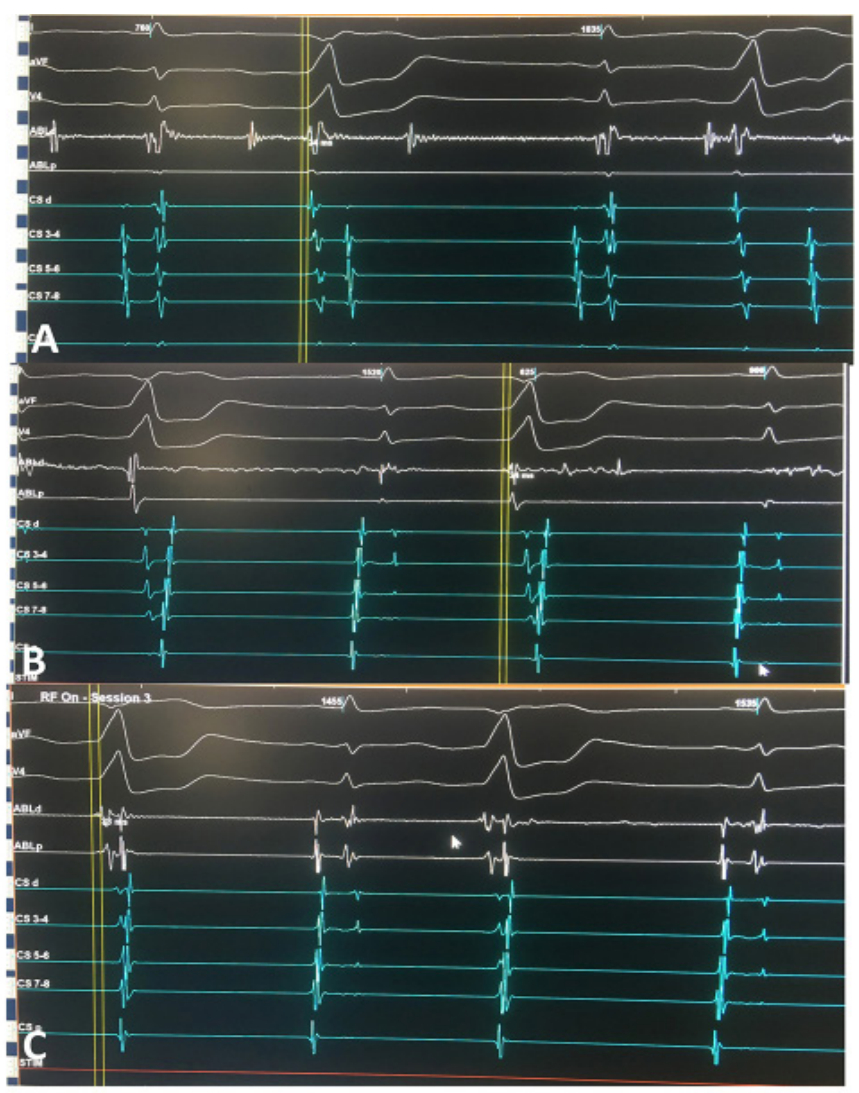

the presence of epicardial fat in this location, radiofrequency catheter ablation (RFCA) of VAs originating from the LVS is quietly challenging. ${ }^{(3)}$ In case of failed ablation attempt in the endocardial aspect of basal left ventricle (just below the aortic valve), ablation from adjacent structures such as the left coronary cusp, the great cardiac vein - the anterior interventricular cardiac vein junction, and the septal right ventricular outflow tract should be tried as effective alternative approaches, respectively. ${ }^{(4-6)}$ As a last and challenging option, an epicardial approach should be kept in mind in all failed cases. ${ }^{(7)}$

Herein, we report on a 45-year-old man with VAs originated from the LVS who underwent an epicardial ablation.

\section{Case}

A 45-year-old man was referred for catheter ablation of symptomatic idiopathic premature ventricular contractions (PVCs) exhibiting a left bundle branch block and inferior axis QRS morphology (Figure 1). Endocardial activation mapping revealed the earliest ventricular activation at the left ventricular summit site where the local ventricular electrogram consisting of two components connected by a fractionated electrogram preceded the QRS onset by $24 \mathrm{~ms}$ (Figure $2 \mathrm{~A}$ and 3A). Pacing from this site did not produce an excellent pace map. Irrigated radiofrequency current with a target temperature of $45^{\circ} \mathrm{C}$ and maximum power output of $45 \mathrm{~W}$ was delivered for $120 \mathrm{~s}$ at this site, resulting in no interruption of the PVCs but a slight change in the QRS morphology, prolongation of the QRS duration, and appearance of pseudo-delta waves during the

Figure 1 .

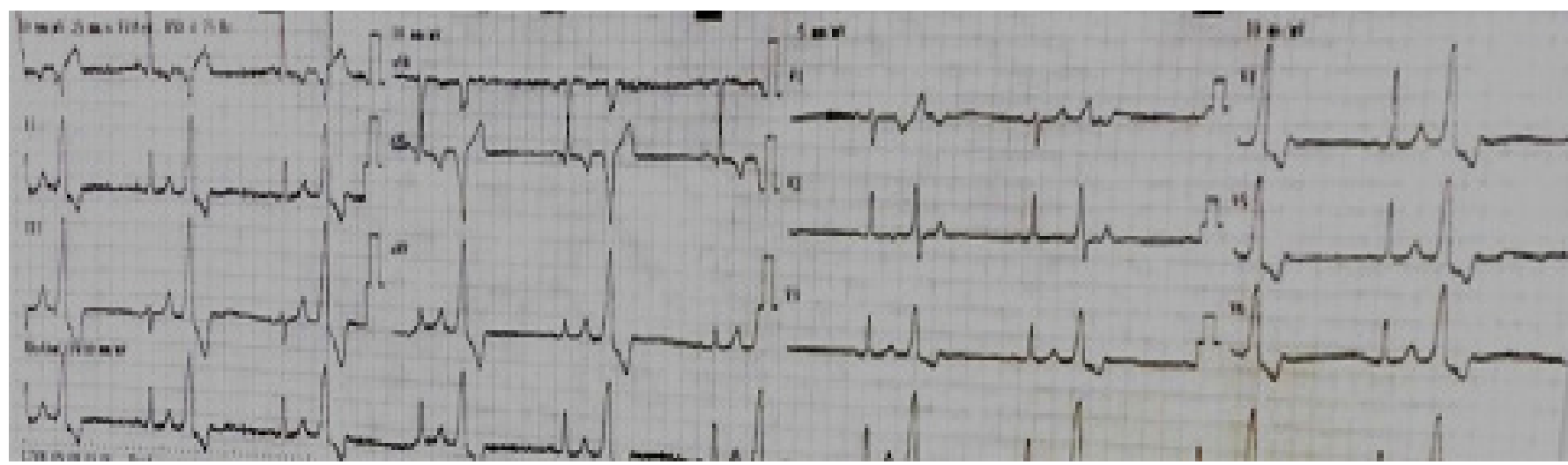


PVCs. Then, the right ventricular outflow tract was mapped. The local ventricular electrogram consisting of two components connected by a fractionated electrogram preceded the QRS onset by $24 \mathrm{~ms}$, again (Figure

Figure 3. A,B

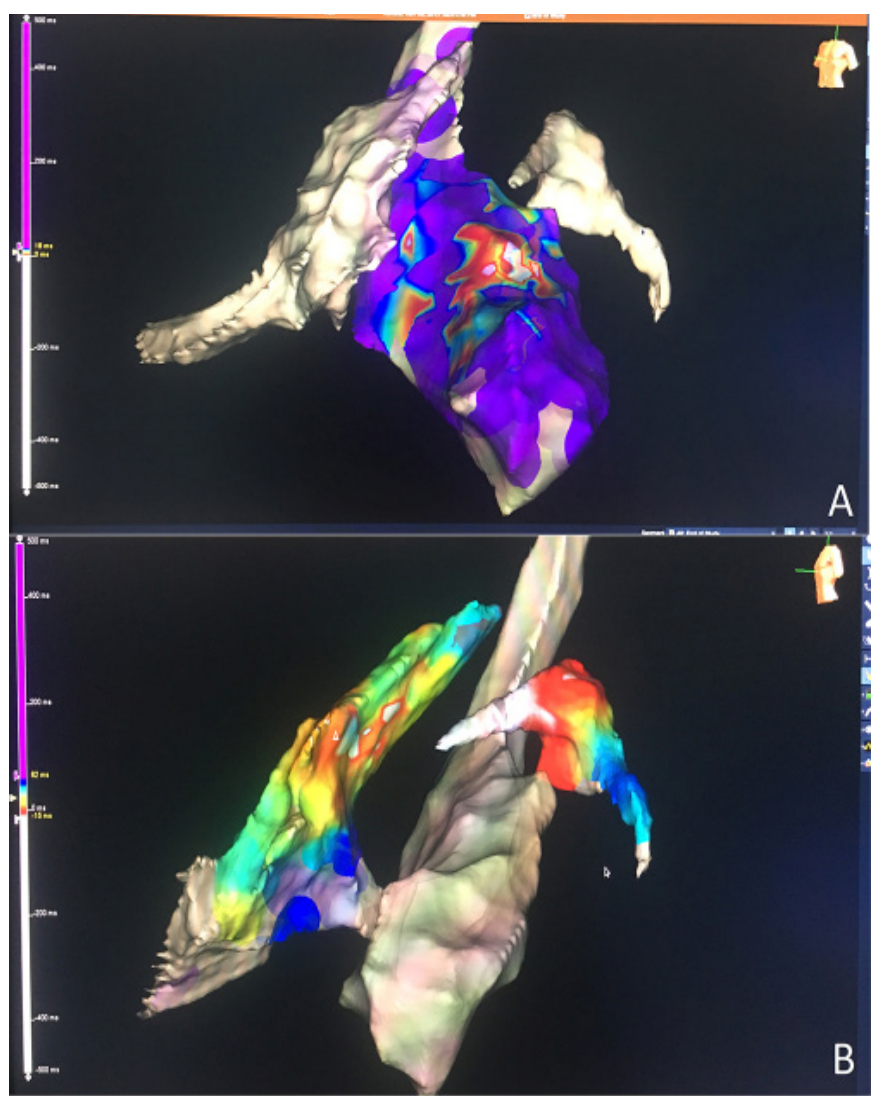

2B and 3B). We did not apply radiofrequency ablation in that site. Lastly, a deflectable 8-F mapping/ablation catheter that had a $3.5-\mathrm{mm}$ irrigated tip (Thermocool, Biosense Webster, Diamond Bar, CA) was advanced to coronary venous system at the great cardiac vein - the anterior interventricular cardiac vein junction. The local ventricular electrogram preceded the QRS onset by $33 \mathrm{~ms}$ (Figure 2C and 3B). Irrigated radiofrequency current with a target temperature of $45^{\circ} \mathrm{C}$ and maximum power output of $35 \mathrm{~W}$ was delivered for $120 \mathrm{~s}$ at this site, resulting in no interruption of the PVCs. Therefore, we decided to perform epicardial puncture.

Epicardial mapping via the subxiphoid pericardial approach was then performed using an irrigated ablation catheter and it revealed the earliest ventricular activation at the site adjacent to the prior endocardial ablation site (Figure 4). At this site, the local ventricular electrogram was similar in morphology to that recorded from the endocardial ablation site and preceded the QRS onset by $48 \mathrm{~ms}$. Pacing from this site produced an excellent match to the QRS complex of the changed PVCs. Left coronary artery angiography revealed that this site was located on the right and upper side of the left anterior descending coronary artery and more than $2 \mathrm{~cm}$ away from that artery (Figure 5). A single application of irrigated radiofrequency current in the

Figure 4.

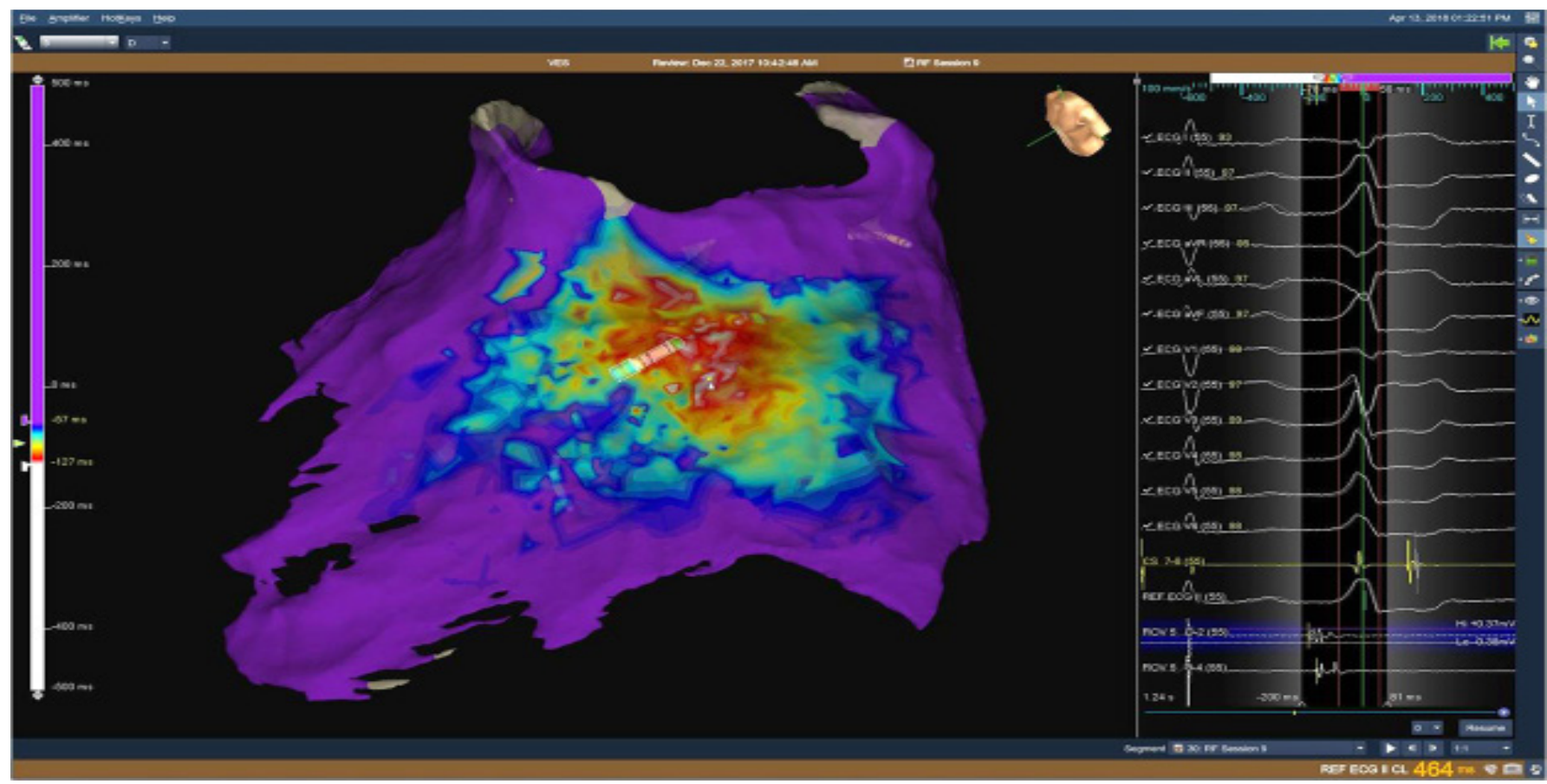


power-control mode at $30 \mathrm{~W}$ delivered for $60 \mathrm{~s}$ at this site eliminated the PVCs (Figure 6). No complications occurred.

During a follow-up of more than 6 months, the patient has not experienced any further palpitations and no VA has been documented on his Holter recordings.

\section{Discussion}

Catheter ablation of VAs originating from the LVS can be challenging, due to close the proximity to critical anatomic structures such as major coronary vessels. Furthermore, thick epicardial fat may cause inability to deliver effective radiofrequency lesions..$^{(3,7,8)}$ To deal with these anatomic obstacles, radiofrequency application from adjacent sites, such as the left coronary cusp region or coronary venous system may be used after failed left ventricular endocardial ablation attempts. ${ }^{(4-6)}$

Figure 5.

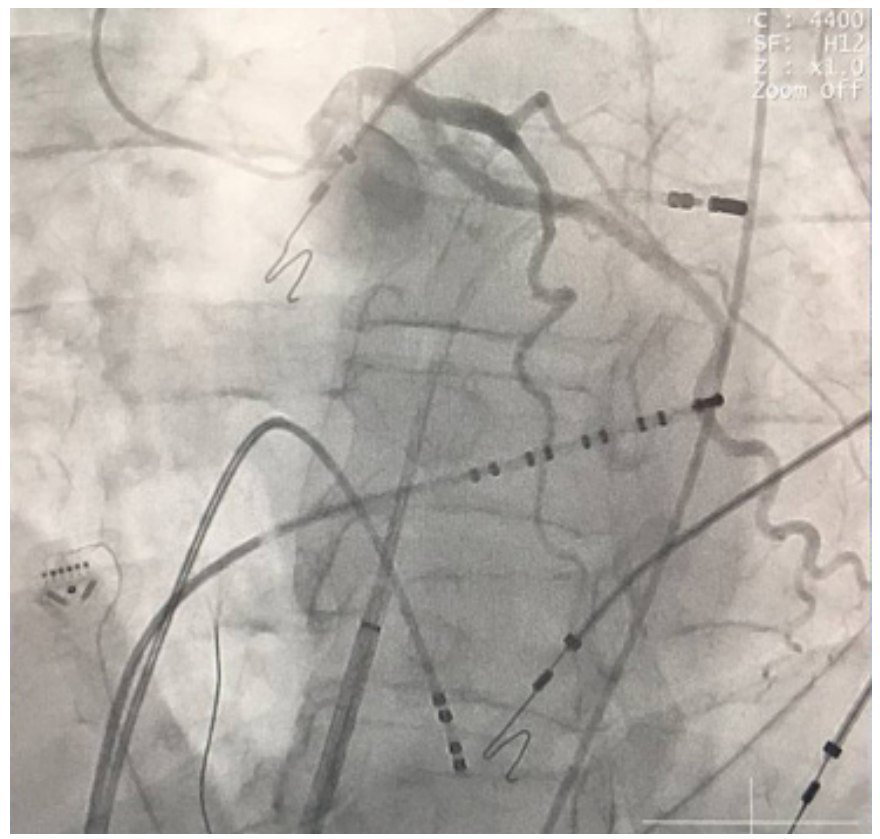

Such a stepwise approach is mandatory in case of VAs originating from the LVS to achieve success. Proper evaluation of intracardiac electrograms together with 3-dimensional electroanatomic mapping is especially helpful in defining the best ablation site.

In our case, we used a stepwise approach to find the earliest site. We firstly mapped the left ventricle based on ECG morphology suggesting the left ventricular origin. Then, coronary cusps, the right ventricle, and coronary venous system mapped, respectively. After ablation from adjacent sites failed, we considered a percutaneous epicardial access for mapping and ablation. It should be kept in mind that, in about two-thirds of cases, radiofrequency delivery in the epicardial location might be aborted because of proximity to major epicardial coronary vessels (7). However, in the current case, we verified safe distance from major coronary vessels by using coronary angiography. In our case, to demonstrate whether VA originates from typical LVS region additional scopic views such as left anterior oblique and right anterior oblique should have been used.

Another possible dilemma during RFCA in the LVS region is that the presence of epicardial fat at the tissuecatheter interface may reduce ablation success. It was previously demonstrated that inadequate lesions by radiofrequency energy due to epicardial fat may be overcome with a different source of ablation energy, such as cryoablation. ${ }^{(9)}$ In rare cases, video-assisted thoracoscopy guided minimally invasive surgical ablation may be used. ${ }^{(10)}$

In patients with VAs originating from the LV summit, the outcome of ablation from both endocardial and epicardial sites may be poor. A stepwise approach to find the earliest activation site is mandatory to achieve success.

Figure 6.

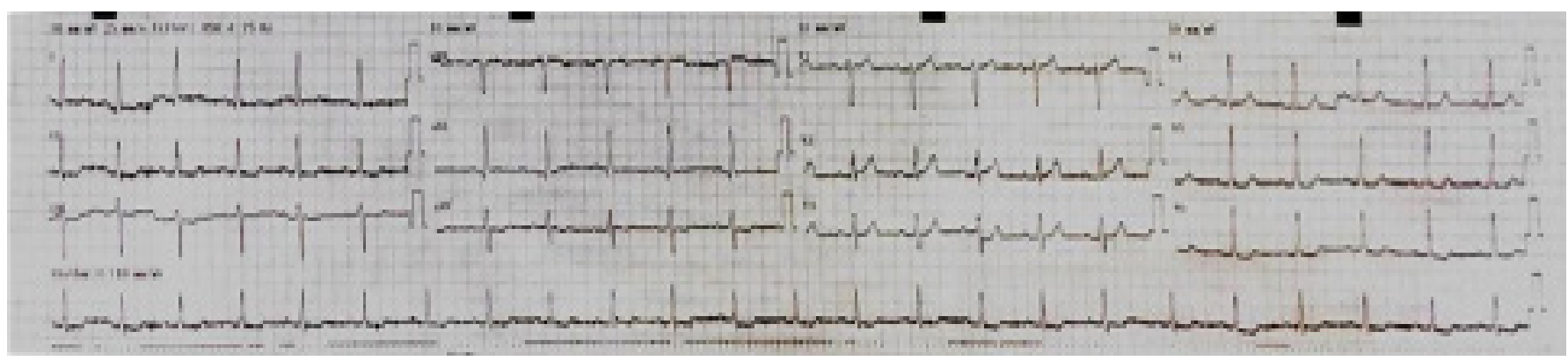


References

1. Stevenson WG, Soejima K. Catheter ablation for ventricular tachycardia. Circulation. 2007;115:2750-2760. DOI: 10.1161/CIRCULATIONAHA. 106.655720

2. Mc Alpine WA. Heart and CoronaryArteries. New York: Springer-Verlag; 1975.

3. Yamada T, McElderry HT, Doppalapudi H, Okada T, Murakami Y, Yoshida Y, Yoshida N, Inden Y, Murohara T, Plumb VJ, Kay GN. Idiopathic ventricular arrhythmias originating from the leftventricular summit: anatomic concepts relevant to ablation. CircArrhythmElectrophysiol. 2010;3:616-623. doi: 10.1161/CIRCEP.110.939744.

4. Jauregui Abularach ME, Campos B, Park KM, Tschabrunn CM, Frankel DS, Park RE, Gerstenfeld EP, Mountantonakis SE, Mountantonakis S, Garcia FC, Dixit S, Tzou WS, Hutchinson MD, Lin D, Riley MP, Cooper JM, Bala R, Callans DJ, Marchlinski FE. Ablation of ventricula rarrhythmias arising near the anterior epicardial veins from the left sinus of Valsalva region: ECG features, anatomic distance, and outcome. HeartRhythm. 2012;9:865-873. doi: 10.1016/j.hrthm.2012.01.022.

5. Obel OA, d'Avila A, Neuzil P, Saad EB, Ruskin JN, Reddy VY. Ablation of left ventricular epicardial outflow tract tachycardia from the distal great cardiac vein. J AmCollCardiol. 2006;48:1813-1817. doi: 10.1016/j.jacc.2006.06.006.

6. Frankel DS, Mountantonakis S, Dahu MI, Marchlinski FE. Elimination of ventricular arrhythmias originating from the anterior interventricular vein with ablation in the right ventricular outflow tract. HeartRhythm. 2014;11:S217.

7. Santangeli P, Marchlinski FE, Zado ES, Benhayon D, Hutchinson MD, Lin D, Frankel DS, Riley MP, Supple GE, Garcia FC, Bala R, Desjardins B, Callans DJ, Dixit S. Percutaneous epicardial ablation of ventricular arrhythmias arising from the left ventricular summit: outcomes and electrocardiogram correlates of success. CircArrhythmElectrophysiol. 2015 Apr;8(2):337-43. doi: 10.1161/CIRCEP.114.002377.

8. Yamada T, McElderry HT, Okada T, Murakami Y, Doppalapudi H, Yoshida N, Yoshida Y, Inden Y, Murohara T, Epstein AE, Plumb VJ, Kay GN. Idiopathic left ventricular arrhythmias originating adjacent to the left aortic sinus of valsalva: electrophysiological rationale for the surface electrocardiogram. J CardiovascElectrophysiol. 2010;21:170-176. doi: 10.1111/j.1540-8167.2009.01608.x.

9. DiBiase L, Al-Ahamad A, Santangeli P, Hsia HH, Sanchez J, Bai R, Bailey S, Horton R, Gallinghouse GJ, Burkhardt DJ, Lakkireddy D, Yang Y, Badhwar N, Scheinman M, Tung R, DelloRusso A, Pelargonio G, Casella M, Tomassoni G, Shivkumar K, Natale A. Safety and outcomes of cryoablation for ventricular tachyarrhythmias: results from a multicenterexperience. HeartRhythm. 2011;8:968-974. doi: 10.1016/j. hrthm.2011.02.038.

10. Aksu T, Erdem Guler T, Yalin K.Successfulablation of an epicardial ventricular tachycardia by video-assisted thoracoscopy.Europace. 2015 Jul;17(7):1116. doi: 10.1093/europace/euv012.

Received: $17 / 05 / 2018$

Accepted: 06/06/2018

Published: 15/09/2018

Disclosure and conflicts of interest:

The authors declare no conflict of interest.

\section{Corresponding author:}

Dr. Tolga Aksu

Mail: aksutolga@gmail.com

Aksu T., Güler T. E., Bozyel S.: Idiopathic Premature Ventricular Contractions Originating From Left Ventricular Summit Successfully Ablated From The Epicardial Approach EJCM 2018; 06 (3): 117-121. Doi: 10.32596/ejcm.18.00317. 